\section{A parable for regenerative medicine}

In this ongoing parable of regenerative medicine, the initial hope was that we were in an advantageous position to take a "shot on goal" by moving quickly to clinical studies. The rationale was noble, clear, and compelling, particularly given the unmet clinical need and the robust results of early scientific studies. However, it now is becoming increasingly clear that we may not have the optimal cell type in hand, let alone a clear understanding of other key variables such as in vivo delivery, efficiency of grafting, and suppression of alternative, unwanted cell phenotypes (e.g., pacemaker cell formation in the midst of cardiac muscle regeneration). Cardiovascular stem cell biology still remains one of the most intriguing fields of scientific inquiry in the cardiovascular field and holds great long-term potential. Perhaps, given our growing understanding of the complexity of cell therapy for heart disease, the time has come to "move the ball down the field" by first trying to understand the mechanistic basis of this potential therapy.

Address correspondence to: Kenneth R. Chien, MGH Cardiovascular Research Center, Massachusetts General Hospital,
Richard B. Simches Research Center/CPZN 3208, 185 Cambridge Street, Boston, Massachusetts 02114-2790, USA. Phone: (617) 643-3440; Fax: (617) 643-3451: E-mail: kchien@partners.org.

1. Assmus, B., et al. 2002. Transplantation of Progenitor Cells and Regeneration Enhancement in Acute Myocardial Infarction (TOPCARE-AMI). Circulation. 106:3009-3017.

2. Meyer, G.P., et al. 2006. Intracoronary bone marrow cell transfer after myocardial infarction: eighteen months' follow-up data from the randomized, controlled BOOST (BOne marrOw transfer to enhance ST-elevation infarct regeneration) trial. Circulation. 113:1287-1294.

3. Janssens, S., et al. 2006. Autologous bone marrow-derived stem-cell transfer in patients with ST-segment elevation myocardial infarction: double-blind, randomised controlled trial. Lancet. 367:113-121.

4. Lunde, K., et al. 2005. Autologous stem cell transplantation in acute myocardial infarction: the ASTAMI randomized controlled trial. Intracoronary transplantation of autologous mononuclear bone marrow cells, study design and safety aspects. Scand. Cardiovasc. J. 39:150-158.

5. Zohlnhofer, D., et al. 2006. Stem cell mobilization by granulocyte colony-stimulating factor in patients with acute myocardial infarction: a randomized controlled trial. JAMA. 295:1003-1010.

6. Cleland, J.G., Freemantle, N., Coletta, A.P., and Clark, A.L. 2006. Clinical trials update from the American Heart Association: REPAIR-AMI, ASTAMI, JELIS, MEGA, REVIVE-II, SURVIVE, and PROACTIVE. Eur. J. Heart Fail. 8:105-110.
7. Wollert, K.C., and Drexler, H. 2006. Cell-based therapy for heart failure. Curr. Opin. Cardiol. 21:234-239.

8. Orlic, D., et al. 2001. Mobilized bone marrow cells repair the infarcted heart, improving function and survival. Proc. Natl. Acad. Sci. U. S. A. 98:10344-10349.

9. Orlic, D., et al. 2001. Bone marrow cells regenerate infarcted myocardium. Nature. 410:701-705.

10. Hofmann, M., et al. 2005. Monitoring of bone marrow cell homing into the infarcted human myocardium. Circulation. 111:2198-2202.

11. Murry, C.E., et al. 2004. Haematopoietic stem cells do not transdifferentiate into cardiac myocytes in myocardial infarcts. Nature. 428:664-668.

12. Balsam, L.B., et al. 2004. Haematopoietic stem cells adopt mature haematopoietic fates in ischaemic myocardium. Nature. 428:668-673.

13. Nygren, J.M., et al. 2004. Bone marrow-derived hematopoietic cells generate cardiomyocytes at a low frequency through cell fusion, but not transdifferentiation. Nat. Med. 10:494-501.

14. Dimmeler, S., Zeiher, A.M., and Schneider, M.D. 2005. Unchain my heart: the scientific foundations of cardiac repair. J. Clin. Invest. 115:572-583. doi:10.1172/JCI200524283

15. Chien, K.R. 2004. Stem cells: lost in translation. Nature. 428:607-608.

16. Fazel, S., et al. 2006. Cardioprotective c-kit ${ }^{+}$cells are from the bone marrow and regulate the myocardial balance of angiogenic cytokines. J. Clin. Invest. 116:1865-1877. doi:10.1172/JCI27019.

17. Beltrami, A.P., et al. 2003. Adult cardiac stem cells are multipotent and support myocardial regeneration. Cell. 114:763-776.

18. Mansour, S., et al. 2006. Intracoronary delivery of hematopoietic bone marrow stem cells and luminal loss of the infarct-related artery in patients with recent myocardial infarction. J. Am. Coll. Cardiol. 47:1727-1730.

\title{
The leak stops here: platelets as delivery vehicles for coagulation factors
}

\author{
Katherine A. High \\ Howard Hughes Medical Institute, The Children's Hospital of Philadelphia, Philadelphia, Pennsylvania, USA.
}

\begin{abstract}
Gene therapy is an attractive approach for the treatment of hemophilia, as continuous expression of donated clotting factor VIII (FVIII) DNA would ensure clotting factor replacement at constant circulating levels rather than at the peaks and troughs that characterize the current protein infusion therapeutic approach. In this issue of the JCI, Shi et al. describe an interesting variant of a gene transfer approach for hemophilia (see the related article beginning on page 1974). They show that targeted expression of FVIII in megakaryocytes, with storage in the $\alpha$-granules of platelets, has the advantage of delivering clotting factors directly to the site of an injury, where platelets accumulate in large numbers and undergo activation accompanied by release of granule contents. Earlier clinical experience with gene transfer into hematopoietic cells highlighted the potential safety risks of this approach, but an $F 8$ transgene may represent a lower risk than transgenes for growth factors or their receptors.
\end{abstract}

Nonstandard abbreviations used: FVIII, factor VIII. Conflict of interest: The author holds issued patents related to the use of adeno-associated virus for the treatment of hemophilia.

Citation for this article: J. Clin. Invest. 116:1840-1842 (2006). doi:10.1172/JCI29193.
Gene transfer for the treatment of genetic disease remains one of the most compelling ideas in modern molecular medicine. In genetic disease, where the therapeutic objective is long-term expression of a specific protein, there are - broadly speaking - two potential strategies: gene transfer into a stem cell via an integrating vector, so that all daughter cells contain the genetic modification, and gene transfer with a nonintegrating vector into long-lived postmitotic cells (such as skeletal or cardiac muscle, hepatocytes, or cells in the central nervous system), so that long-lasting expression is achieved even without vector integration. Both of these strategies have been used to achieve long-term expression of a clotting factor, with phenotypic correction, in large animal models of hemophilia, one by use of a retroviral vector in neonatal (rapidly dividing) hepatocytes (1), and the other by use of an adeno-associated virus vector introduced into mature hepatocytes (2-4) (Figure 1A).

One of the advantages of hemophilia as a model for gene therapy is that tissue-specific expression of the donated gene is not 
A

Introduction of retroviral F9 integrating vector into rapidly proliferating neonatal hepatocytes
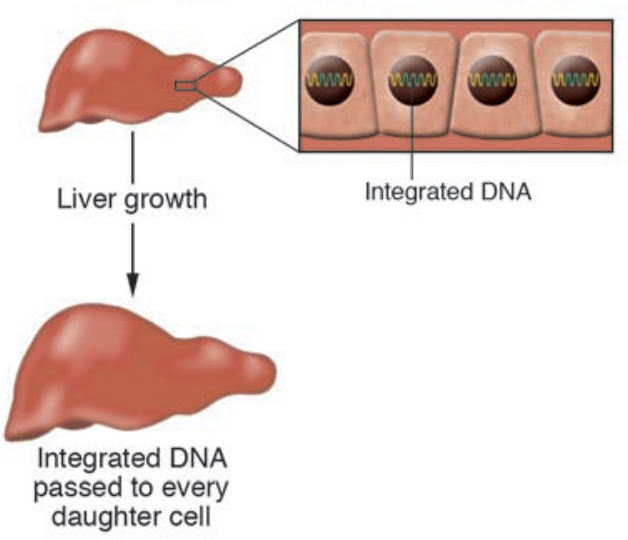

B

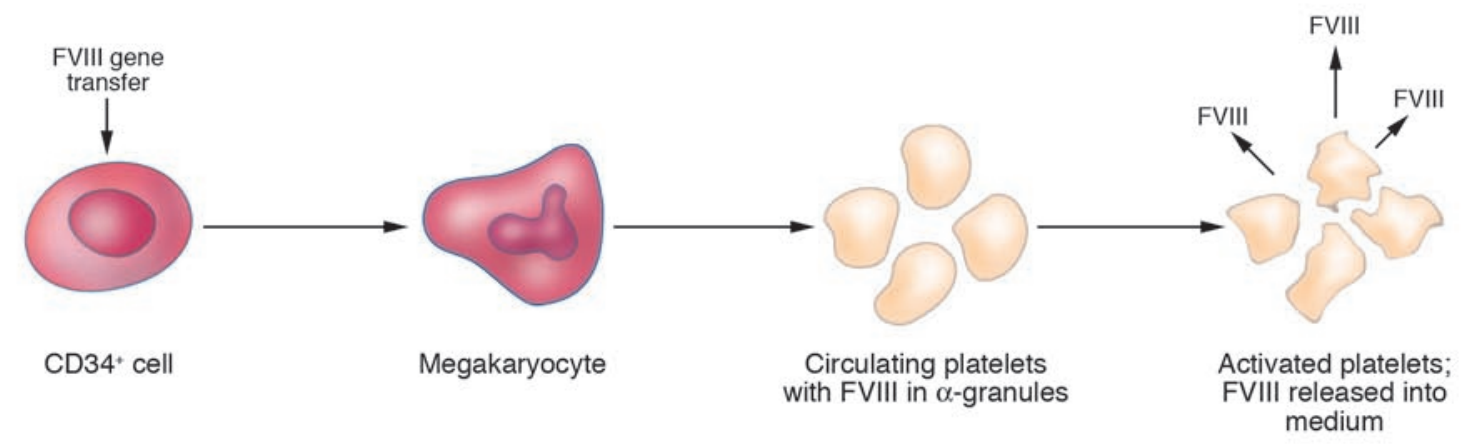

Figure 1

Strategies for achieving long-term protein expression in genetic disease. (A) Gene transfer strategies leading to long-term protein expression in canine hemophilia. At left, intravenous infusion of a retroviral vector expressing canine factor IX (FIX) into neonatal dogs results in transduction of hepatocytes with transmission of the integrated DNA into all daughter cells and continuous expression of factor IX in the growing animal (1). In the approach shown to the right, with portal vein infusion of adeno-associated virus-FVIII (AAV-FVIII) or -factor IX into an adult animal, the donated DNA is stabilized in an episomal form, with long-term expression of the clotting factor (2-4). (B) After gene transfer into hematopoietic stem cells (CD34+ cells), megakaryocytes carrying the donated gene give rise to platelets that contain FVIII packaged in the $\alpha$-granules. Activation of platelets at the site of an injury leads to release of granule contents, including FVIII.

required. Although clotting factors are normally made in the liver (in both hepatocytes and endothelial cells), biologically active coagulation factors can be synthesized in a variety of tissues; in fact, some of the currently marketed recombinant clotting factors are synthesized in baby hamster kidney and $\mathrm{CHO}$ cells. This latitude in choice of target tissue has been amply exploited in the field, and cures of murine models of hemophilia have been achieved through clotting factor expression in skeletal muscle (5), the epidermis (6), and bone marrow stromal cells (7).

\section{Factor VIII gene expression in megakaryocytes - a therapeutic platelet?}

In 2003, Poncz and coworkers proposed and demonstrated the feasibility of gene transfer into platelet precursors as a strategy for the treatment of hemophilia A, an X-linked disorder caused by deficiency of coagulation factor VIII (FVIII; Figure 1B) (8). Using the platelet-specific glycoprotein $1 \mathrm{~b} \alpha$ promoter, they constructed a transgenic mouse expressing human B-domain-deleted FVIII and showed that expression of FVIII in megakaryocytes and platelets improved hemostasis in hemophilia A mice (as judged by bleeding times), even though circulating FVIII levels did not rise above $1 \%$ of normal. They further showed that human FVIII colocalized with VWF in the $\alpha$-granules of platelets and that transfusion of platelets from the transgenic mice into mice with hemophilia A resulted in an improvement in hemostasis in these animals as well. That hemostasis improved even without an increase in circulating levels of FVIII was due to platelet activation and subsequent release of granule contents, including FVIII, which was demonstrated by measuring FVIII levels in a platelet lysate. It was suggested that local release of FVIII from the activated platelet at the site of a bleed could result in effective hemostasis even in the presence of circulating antibodies (i.e., inhibitors) to FVIII. In this issue of the JCI, Shi and colleagues (9) provide convincing evidence in support of this approach and show for the first time to my knowledge that this strategy improves hemostasis even in the presence of high-titer inhibitors to FVIII, a problem encountered in as many as $25-30 \%$ of patients with hemophilia A.

\section{Feasibility issues: scaling up and engraftment}

Can this encouraging result in mice be extended to humans with the disease? An advantage for hemophilia in assessing the likelihood of 
treatment success in humans is the availability of a large animal model of the disease. The availability of strains of hemophilic dogs that are prone to FVIII inhibitor formation (10), of the cloned canine F8 cDNA (11), and of well-established techniques for bone marrow transplantation of genetically modified cells in dogs (12) means that the feasibility of this strategy can be readily assessed in an organism close in size to humans. If feasibility is demonstrated in the hemophilic dog model, it will still be critical to assess the safety of the approach. Transduction of CD34+ hematopoietic stem cells with an integrating vector has already been used successfully to treat lymphoid immunodeficiency disorders in children with X-linked SCID (13) and adenosine deaminase deficiency SCID (ADA-SCID) (14). In these disorders, the success of the gene transfer approach was dependent on a selective survival and growth advantage for the transduced cells, which eventually came to represent a substantial proportion of the circulating cells. In the case of FVIII, of course, no survival advantage accrues to the transduced cells, so the powerful in vivo selection that is a key to success in immunodeficiency disorders plays no role here. Potential strategies to circumvent this obstacle include full myeloablation prior to transplantation with genetically modified autologous cells so that the repopulated marrow contains mostly genetically modified cells (as essentially used in these experiments, in which mice were lethally irradiated prior to transplant), or coexpression of the donated gene with a drug resistance marker (15) so that transplantation of genetically modified autologous cells, followed by drug selection, results in a strong in vivo survival advantage for the transduced cells. Since short-term risks of autologous transplantation are very low and the presence of both hemophilic and acquired inhibitors can be a difficult problem in treatment management, the risk/benefit ratio for gene transfer in this setting may well be a favorable one.

\section{Safety issues: integration and insertional mutagenesis}

Aside from issues related to engraftment and survival of genetically modified cells, though, the use of integrating vectors raises the concern of insertional mutagenesis. This had been an anticipated complication of gene transfer with integrating vectors, but had remained largely theoretical until 2002, when it was first reported in two children three years after they had undergone successful gene transfer for X-linked SCID $(16,17)$. In incisive and compelling work, the investigators that first described this complication demonstrated progressive outgrowth of a leukemic clone in which the donated gene had integrated within the LMO2 oncogene. A recent study suggests that the identity of the donated gene itself (in the case of X-linked SCID, a receptor for a cytokine growth factor) contributed to the adverse event (18). The absence of such complications in ADA-SCID, in which the donated gene is an enzyme rather than a growth factor receptor, is consistent with a role for the transgene product and suggests that genes like F8 (a cofactor for an enzyme) would be unlikely to lead to the complication seen in X-linked SCID. An emerging concept is that growth factors, their receptors, and signal transduction molecules may represent a higher level of risk as transgene products compared with enzymes and cofactors. Proposed strategies for avoiding risks related to insertional mutagenesis include use of insulating elements to "shield" the donated gene from effects of flanking DNA and vice versa (19), development of vectors that target certain "safe" sites in the chromosomal DNA (20), and use of suicide elements (e.g., a thymidine kinase gene) within the donated gene cassette so that a rapidly expanding clone can be controlled with a small-molecule drug (e.g., gancyclovir) (21).

None of these concerns represent insuperable obstacles, and the work described by Shi et al. (9) may herald an important therapeutic advance for those individuals with hemophilic or acquired inhibitors. Particularly for those in the latter group, where mortality within the first year after diagnosis can be as high as $10 \%$, a strategy based on infusion of genetically modified autologous bone marrow cells may be a reasonable alternative to current immunosuppressive regimens, which have had only modest success rates.

The availability of differing gene transfer approaches for hemophilia will be important for this patient group, in which - for example - some patients can never be candidates for liver-directed gene transfer strategies due to underlying liver disease. In the long term, it is likely that there will be multiple successful strategies for gene transfer in hemophilia. An approach that generates "therapeutic" platelets would be a welcome advance.

Address correspondence to: Katherine A. High, The Children's Hospital of Philadelphia, 3615 Civic Center Boulevard, Philadelphia, Pennsylvania 19104, USA. Phone: (215) 590-4521; Fax: (215) 590-3660; E-mail: high@email.chop.edu.
1. Xu, L., et al. 2003. Neonatal or hepatocyte growth factor-potentiated adult gene therapy with a retroviral vector results in therapeutic levels of canine factor IX for hemophilia B. Blood. 101:3924-3932.

2. Mount, J.D., et al. 2002. Sustained phenotypic correction of hemophilia B dogs with a factor IX null mutation by liver-directed gene therapy. Blood. 99:2670-2676.

3. Scallan, C.D., et al. 2003. Sustained phenotypic correction of canine hemophilia A using an adenoassociated viral vector. Blood. 102:2031-2037.

4. Jiang, H., et al. 2006. Multi-year therapeutic benefit of AAV serotypes 2, 6 and 8 delivering factor VIII to hemophilia A mice and dogs. Blood. doi:10.1182/ blood-2005-12-5115.

5. Herzog, R.W., et al. 1997. Stable gene transfer and expression of human blood coagulation factor IX after intramuscular injection of recombinant adeno-associated virus. Proc. Natl. Acad. Sci. U. S. A. 94:5804-5809.

6. Fakharzadeh, S.S., Zhang, Y., Sarkar, R., and Kazazian, H.H., Jr. 2000. Correction of the coagulation defect in hemophilia A mice through factor VIII expression in skin. Blood. 95:2799-2805.

7. Chuah, M.K., et al. 2000. Long-term persistence of human bone marrow stromal cells transduced with factor VIII-retroviral vectors and transient production of therapeutic levels of human factor VIII in nonmyeloablated immunodeficient mice. Hum. Gene Ther. 11:729-738.

8. Yarovoi, H.V., et al. 2003. Factor VIII ectopically expressed in platelets: efficacy in hemophilia A treatment. Blood. 102:4006-4013.

9. Shi, Q., et al. 2006. Factor VIII ectopically targeted to platelets is therapeutic in hemophilia A with high-titer inhibitory antibodies. J. Clin. Invest. 116:1974-1982. doi:10.1172/JCI28416.

10. Rawle, F.E., and Lillicrap, D. 2004. Preclinical animal models for hemophilia gene therapy: predictive value and limitations. Semin. Thromb. Hemost. 30:205-213.

11. Cameron, C., et al. 1998. The canine factor VIII cDNA and 5' flanking sequence. Thromb. Haemost. 79:317-322.

12. Neff, T., Beard, B.C., and Kiem, H.P. 2006. Survival of the fittest: in vivo selection and stem cell gene therapy. Blood. 107:1751-1760.

13. Cavazzana-Calvo, M., et al. 2000. Gene therapy of human severe combined immunodeficiency (SCID)-X1 disease. Science. 288:669-672.

14. Aiuti, A., et al. 2002. Correction of ADA-SCID by stem cell gene therapy combined with nonmyeloablative conditioning. Science. 296:2410-2413.

15. Zielske, S.P., Reese, J.S., Lingas, K.T., Donze, J.R., and Gerson, S.L. 2003. In vivo selection of MGMT(P140K) lentivirus-transduced human NOD/SCID repopulating cells without pretransplant irradiation conditioning. J. Clin. Invest. 112:1561-1570. doi:10.1172/JCI200317922.

16. Hacein-Bey-Abina, S., et al. 2003. A serious adverse event after successful gene therapy for X-linked severe combined immunodeficiency. N. Engl.J. Med. 348:255-256.

17. Hacein-Bey-Abina, S., et al. 2003. LMO2-associated clonal $\mathrm{T}$ cell proliferation in two patients after gene therapy for SCID-X1. Science. 302:415-419.

18. Woods, N.B., Bottero, V., Schmidt, M., von Kalle, C., and Verma, I.M. 2006. Gene therapy: therapeutic gene causing lymphoma. Nature. 440:1123.

19. Chung, J.H., Bell, A.C., and Felsenfeld, G. 1997. Characterization of the chicken beta-globin insulator. Proc. Natl. Acad. Sci. U. S. A. 94:575-580.

20. Chalberg, T.W., et al. 2006. Integration specificity of phage phiC31 integrase in the human genome. J. Mol. Biol. 357:28-48.

21. Springer, C.J., and Niculescu-Duvaz, I. 2000. Prodrug-activating systems in suicide gene therapy. J. Clin. Invest. 105:1161-1167. 